\title{
EROSION AND DEPOSITION MECHANISMS IN FUSION PLASMAS
}

\author{
A. Kirschner \\ Institut für Energie- und Klimaforschung - Plasmaphysik, Forschungszentrum Jülich GmbH, \\ Association EURATOM-FZJ, Trilateral Euregio Cluster, 52425 Jülich, Germany
}

\begin{abstract}
An overview of erosion and deposition processes in fusion machines is presented. The underlying physical and chemical mechanisms are explained. The impact of erosion and deposition on wall lifetime and tritium retention, which define the availability of future fusion machines such as ITER, is discussed. Also, examples of erosion and deposition observed in present fusion experiments are presented.
\end{abstract}

\section{INTRODUCTION}

The next major step on the way to a fusion reactor is the international experimental reactor ITER ${ }^{1}$. In longpulse (about 400s) or even steady-state operation, which both are foreseen for ITER, erosion and deposition processes become more crucial than in current fusion experiments. Erosion of wall material leads to limitation of the lifetime of the wall components. Whereas on the one hand deposition of eroded material can eventually reduce net erosion, it will lead to formation of deposited layers on the other hand. Main concern of deposited material is its ability to retain large amounts of fuel, which in ITER will consist of 50\% deuterium and 50\% tritium within the active phase of operation. The invessel retention of radioactive tritium will be limited due to safety regulations. Current estimations of wall lifetime and tritium retention for ITER are based on extrapolations from present experiments or modeling calculations and imply relatively large uncertainties ${ }^{2,3}$. Nevertheless, they indicate that the number of pulses before reaching the tritium retention limit or the maximum allowed erosion of wall components could be unacceptably low for an economical operation. From this, it is obvious that both erosion and deposition of wall material will strongly determine the availability of ITER. It is therefore necessary to understand the involved mechanisms and to find possibilities to minimize erosion and deposition.

The erosion and deposition properties naturally depend on the material choice. In ITER there are currently three different materials under discussion for the use as wall cladding. Beryllium $(\mathrm{Be})$ is planned to cover the first wall in the main chamber. Compared to elements of high atomic number $(Z)$ eroded $\mathrm{Be}(Z=4)$ entering the plasma leads to lower plasma cooling due to radiation. With respect to the large area of the first wall the use of low- $Z$ Be is therefore more beneficial although in general the sputtering of low- $Z$ elements is larger than that of high- $Z$ ones. In addition, Be has the advantage of being a good oxygen getter. For the socalled baffles, which cover the region between the main wall and the divertor plates, tungsten is intended to be used. Here, larger ion fluxes (compared to the main wall) and a significant flux of charge exchange neutrals will reach the surface such that the sputtering should be minimized by using a high- $Z$ material. Tungsten in addition has a relatively high melting point of about $3400^{\circ} \mathrm{C}$. Finally, the divertor plates, on which the maximum particle and heat fluxes will occur, were originally planned to be made of carbon fiber composites (CFC). Carbon-based materials can withstand highest heat loads without melting (sublimation at a temperature of about $3800^{\circ} \mathrm{C}$ ). Therefore problems caused by melt layer loss do not occur. However, carbon-based materials suffer from chemical erosion/sputtering by means of formation of volatile hydrocarbons $C_{x} I_{y}$, where "I" represents hydrogen $\mathrm{H}$ or its isotopes deuterium D and tritium T. The deposition of such species leads to formation of tritium-containing layers inducing the problems as addressed above. It was planned to use CFC divertor plates at the beginning of ITER operation in the non-active phase without tritium. For further operation in the active phase it was foreseen to exchange the CFC plates with tungsten ones to minimize tritium retention by co-deposition. At the moment discussions are ongoing to start already in the non-active phase with a tungsten divertor to reduce the overall costs of the ITER project.

Besides erosion of these "pure" elements also mixed layers, which are formed after erosion and re-deposition processes, have to be taken into account. In the mixture of materials currently foreseen for ITER one can expect the formation of carbides $\left(\mathrm{Be}_{2} \mathrm{C}, \mathrm{WC}\right)$ and also alloys with erosion and other physical properties (e.g. melting point) different from the pure elements. 


\section{EROSION MECHANISMS}

\section{The erosion yield $Y$}

To characterize the strength of erosion the yield $\mathrm{Y}$ is defined as ratio of the averaged number of eroded particles and number of incoming projectiles. It is important that not a single projectile is considered but a large amount of projectiles such that the erosion yield represents the erosion probability. The yield can be determined by the flux of eroded particles $\Gamma_{\text {ero }}$ divided by the flux of incoming projectiles $\Gamma_{\text {in }}$ :

$$
\begin{gathered}
\Gamma_{\text {in }}=\frac{\text { number of incoming particles }}{\text { area } \times \text { time }} \\
\Gamma_{\text {ero }}=\frac{\text { number } \text { of eroded particles }}{\text { area } \times \text { time }} \\
Y=\frac{\Gamma_{\text {ero }}}{\Gamma_{\text {in }}}
\end{gathered}
$$

\section{A. Physical sputtering}

\section{A.1. Basic features}

Within the process of physical sputtering, the momentum of incoming projectiles (energetic ions or neutrals) is transferred to surface atoms of the target material via nuclear collisions. If the transferred energy is large enough to overcome the surface binding energy (which is only known for a few materials, therefore it is common to use the heat of sublimation as an estimate), the surface atom can leave the solid and is physically sputtered. Although the first momentum transfer from projectile to target atoms is directed into the surface, subsequent collisions can lead to a momentum transfer which is directed out of the solid surface. Different regimes of collision can be distinguished mainly depending on the projectile energy and mass:

\section{i) Single collision regime}

After one single collision of the projectile with a target atom, the projectile hits a surface target atom. This process particularly occurs for light projectile ions with low impact energies.

\section{ii) Linear cascade regime}

With medium projectile energies (larger than several $10 \mathrm{eV}$ ) a collision cascade is developing in the solid including also the generation of recoil atoms. However, collisions between two moving atoms are rare.

\section{iii) Spike regime}

At high impact energies $(\mathrm{keV}-\mathrm{MeV})$ and high projectile masses the densities of recoils of the collision cascade is increasing. Inside the spike region most atoms are moving, whereby collisions between simultaneously moving particles become important.

Figure 1 illustrates these different regimes. The first two regimes can be described with the binary collision approximation (BCA), which will be discussed in chapter II.A.2. In the spike regime many-body processes have to be taken into account and the heat spike can lead to a local melting of the solid. However, under the conditions of wall materials in fusion experiments the spike regime is less important than the other two regimes.

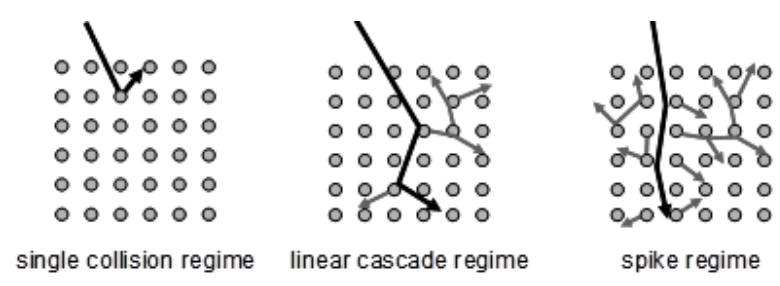

Figure 1: Collision regimes inside a solid induced by impact of a projectile atom.

In general, physical sputtering occurs for all combinations of projectile and target materials. The sputtered species are mostly neutral atoms or small clusters of the target material. Due to the nature of physical sputtering there exists a threshold energy for projectile particles below which the sputtering yield is zero. A surface atom at least has to receive the surface binding energy to be sputtered from the solid.

Besides the impact energy the sputtering yield also depends on the impact angle of projectiles. Also the combination of projectile and substrate material influences the sputter yield. This can be easily understood in terms of the maximum energy transfer factor $\gamma$ for head-on collisions

$$
\gamma=\frac{4 \cdot M_{1} \cdot M_{2}}{\left(M_{1}+M_{2}\right)^{2}}
$$

where $\mathrm{M}_{1}$ and $\mathrm{M}_{2}$ are the masses of projectile and target material respectively. The factor $\gamma$ is maximal $(=1)$ for identical masses of projectile and substrate, $M_{1}=M_{2}$. Physical sputtering does not significantly depend on the surface temperature but is dominated by the kinetics of collisions.

The basics of physical sputtering of single-ion targets, covering theoretical aspects as well as experimental results, are described in ${ }^{4}$. In the following the main dependencies of the sputtering yield are discussed in more detail.

Energy dependence of $Y_{\text {phys }}$

Below the threshold energy $E_{\text {th }}$ the sputter yield is zero. The threshold energy can be estimated for light projectile ions when only two collisions between projectile and solid atoms are involved as shown in figure 1, left part. In the extreme case of head-on collisions the projectile of impact energy $E_{0}$ has the energy $(1-\gamma) \cdot E_{0}$ after reflection at the solid atom. The reflected projectile then can transfer maximum energy of $\gamma \cdot(1-\gamma) \cdot \mathrm{E}_{0}$ to surface atoms of the solid. The sputtered atom finally has an energy of $E_{\text {sputt }}=\gamma \cdot(1-\gamma) \cdot E_{0}-E_{B}$, where $E_{B}$ is the surface binding energy. From this, the threshold energy follows by setting $\mathrm{E}_{\text {sputt }}=0$, thus:

$$
E_{t h}=\frac{E_{B}}{\gamma \cdot(1-\gamma)}
$$


Table 1 summarizes threshold energies for sputtering of beryllium, carbon (low-Z) and tungsten (high-Z) due to deuterium (D) and oxygen (O) as calculated with Eq. (3). As can be seen for sputtering caused by deuterium bombardment, the threshold energy for high- $Z$ materials is significantly larger than for low- $Z$ ones. In addition, the sputtering of high- $Z$ materials due to impurities such as oxygen starts at lower energies than the sputtering due to deuterium. However, Eq. (3) cannot be used universally for calculating threshold energies of physical sputtering. If the masses of projectile and target atoms are similar, wrong threshold energies are delivered (as shown in table 1 for sputtering of beryllium and carbon due to oxygen). This can be easily seen for the extreme case of $M_{1}=M_{2}$ which gives $\gamma=1$ and $E_{\text {th }}$ in Eq. (3) would be infinity. Though, with $M_{1}=M_{2}$ one faces the situation of so-called "self-sputtering", which is a very effective mechanism and cannot be explained with the simple two-collisions model. For the case of $\mathrm{M}_{1} / \mathrm{M}_{2}>0.2$ a fit of experimental data ${ }^{5}$ results in

$$
E_{t h}=8 E_{B} \cdot\left(\frac{M_{1}}{M_{2}}\right)^{2 / 5}
$$

For $\mathrm{M}_{1} / \mathrm{M}_{2}<0.2$ Eq. (3) still is a good approximation of experimental data. The threshold energies according to Eq. (4) for the material combinations discussed so far with $\mathrm{M}_{1} / \mathrm{M}_{2}>0.2$ are added in table 1 in brackets.

Table 1: Threshold energies (eV) for physical sputtering calculated acc. to Eq. (3) and Eq. (4) in brackets.

\begin{tabular}{c|c|c} 
& D & \multicolumn{1}{|c}{ O } \\
\hline $\mathbf{B e}\left(\mathbf{E}_{\mathbf{B}}=\mathbf{3 . 3 8} \mathbf{e V}\right)$ & $14(15)$ & $47(34)$ \\
\hline $\mathbf{C}\left(\mathbf{E}_{\mathbf{B}}=\mathbf{7 . 4 2} \mathbf{e V}\right)$ & $30(-)$ & $373(67)$ \\
\hline $\mathbf{W}\left(\mathbf{E}_{\mathbf{B}}=\mathbf{8 . 8} \mathrm{eV}\right)$ & $214(-)$ & $42(-)$
\end{tabular}

For impact energies above the threshold energy physical sputtering occurs with the sputter yield increasing monotonically until reaching a maximum value at a certain impact energy: more energy can be transferred to surface atoms, which increases the probability for sputtering. Further increase of the impact energy leads to continuous decrease of the sputter yield: the impinging projectiles and therefore also the collision cascades penetrate deeper into the solid and therefore less energy is transferred to surface atoms.

Figure 2 shows as an example the energy dependence of physical sputtering of beryllium due to deuterium at normal incidence calculated with the TRIM ${ }^{6}$ code. More details about the TRIM code will be given in subsection A.2 when discussing the calculation of sputtering yields.

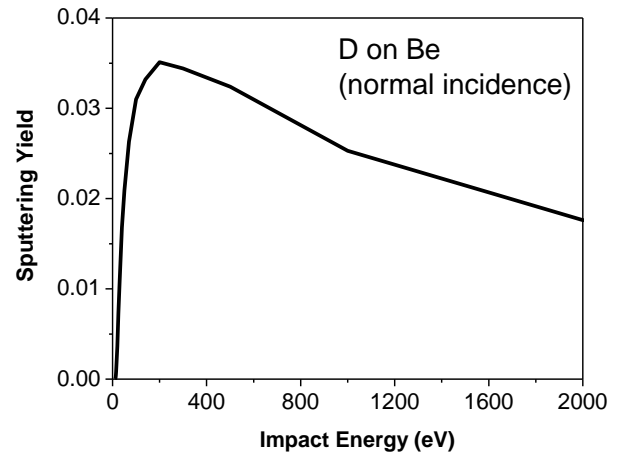

Figure 2: Calculated sputtering yield for $\mathrm{D}$ on $\mathrm{Be}$ in dependence on the impact energy.

It is important to mention that in a plasma, and therefore also in fusion experiments, the impact energy of ions hitting a surface is determined by the ion and electron temperature $\left(T_{i}\right.$ and $\left.T_{e}\right)$, where in many cases

$$
\mathrm{E}_{\mathrm{in}} \sim 3 \cdot \mathrm{Q} \cdot \mathrm{T}_{\mathrm{e}}+2 \cdot \mathrm{T}_{\mathrm{i}}
$$

with Q the charge state of the projectile ${ }^{7}$. The first part of Eq. (5) originates from the acceleration of the ions in the sheath potential and the second part reflects the Maxwell-distributed thermal velocity of the ions and the energy gain in the pre-sheath electric field.

\section{Angular dependence of $Y_{\text {phys }}$}

The angle of incidence $\alpha_{0}$ of impinging projectiles is defined as angle between the velocity vector of the projectile and the surface normal vector. With this definition $\alpha_{0}=0^{\circ}$ represents normal and $\alpha_{0}=90^{\circ}$ grazing incidence. Figure 3 shows the calculated sputtering yield again for deuterium on beryllium but now with a fixed impact energy $E_{0}=200 \mathrm{eV}$ in dependence on the angle of incidence (data from TRIM calculations).

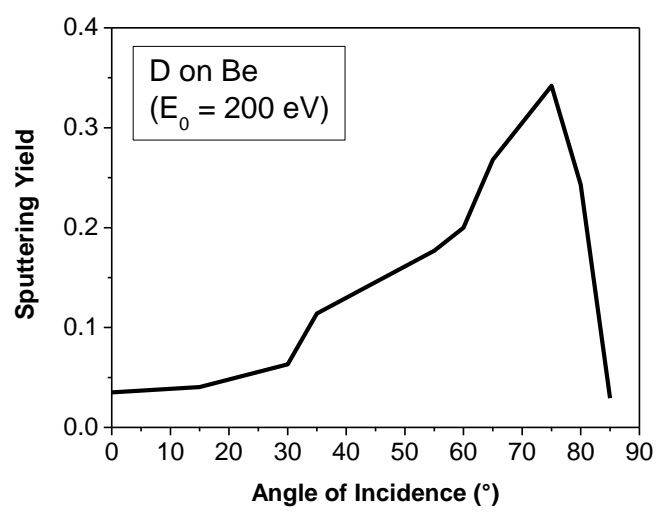

Figure 3: Calculated (TRIM) sputtering yield for D on $\mathrm{Be}$ in dependence on the impact angle.

Starting at normal incidence the sputter yield increases with increasing angle of incidence. With more grazing incidence of the projectiles more energy is deposited near the surface. After reaching a maximum yield (in the example of figure 3 at about $75^{\circ}$ ) the sputtering yield strongly decreases. At theses shallow 
angles reflection of projectiles becomes more important resulting in less energy available at the surface for sputtering. The described angular dependence of physical sputtering assumes smooth (on an atomistic scale) target surfaces. Unpolished surfaces normally exhibit a certain roughness.

Figure 4 demonstrates the effect of surface roughness on the sputter yield on the example of beryllium sputtering due to $300 \mathrm{eV}$ deuterium ions ${ }^{8}$. TRIM simulation assume smooth surface and deviate from measurements at a rough surface especially showing a more pronounced increase with nominal angle of incidence. At rough surfaces two processes have to be taken into account: First, the local angle of incidence differs from the nominal one. Dependent on the nominal angle of incidence one has to consider a distribution of local angles of incidence instead of one fixed angle. Taking e.g. a nominal angle of incidence of $0^{\circ}$, leads to contributions of larger angles in the distribution of local angles of incidence. Thus, at a rough surface the sputter yield will be larger than at a smooth surface taking into account the angular dependence of figure 3. Similarly, at high nominal angles the sputter yield for rough surfaces will be smaller than for smooth ones - especially the maximum yield for a rough surface will be smaller than for a smooth surface. Secondly, sputtered particles can be re-deposited at side walls of valleys on the rough surface. This effect decreases the sputtering yield. Obviously the importance of re-deposition increases with surface roughness and is less important at glancing nominal angles of incidence. Both effects, the distribution of local angles of incidence and redeposition of sputtered particles, are included in the simulation of figure 4 for rough surfaces demonstrating a good agreement with the measured data.

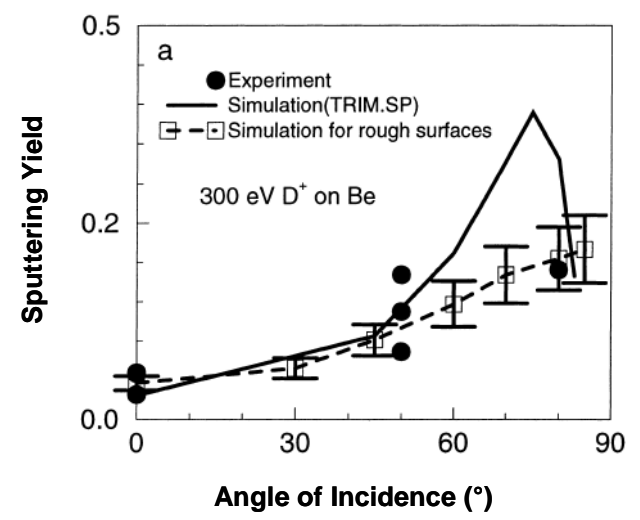

Figure 4: Measured and calculated sputter yields of $\mathrm{D}$ on $\mathrm{Be}$ in dependence on the nominal angle of incidence for a rough surface ${ }^{8}$.

Energy and angular distribution of sputtered particles

In many cases the energy distribution of sputtered particles can be described with a Thompson distribution:

$$
N\left(E_{\text {sputt }}\right) \propto \frac{E_{\text {sputt }}}{\left(E_{\text {sputt }}+E_{B}\right)^{3}}
$$

At $E_{\text {sputt }}=E_{B} / 2$ the energy distribution has a maximum. At higher energies the probability for sputtered particles with the given energy decreases with $1 / E^{2}$. The maximal energy, which can be transferred to sputtered particles equals $E_{\text {sput }}^{\max }=\gamma \cdot(1-\gamma) \cdot E_{0}-E_{B}$ and therefore has to be included in Eq. (6) as cut-off. Measurements of the energy distribution of sputtered particles agree fairly well with Eq. (6) for heavy-ion sputtering at normal incidence in the range of $1 \mathrm{keV}^{9}$, whereas deviations occur for light impact ions and/or non-normal incidence.

The angular distribution of sputtered particles for normal incidence by medium and heavy ions can be approximated with a cosine distribution. This follows from the theory of cascade sputtering with the assumption of an isotropic collision cascade. Deviations to an over-cosine distribution, which peaks towards the surface normal, arise for light-ion bombardment. This deviation tends to be stronger with low impact energies and/or metals with high surface binding energy ${ }^{10}$. In practice surfaces are rough and data of angle distributions are rare, thus a cosine distribution is a good approximation.

\section{A.2. Calculating of physical sputtering yields}

Experimental data on physical sputtering yields are mainly obtained by means of ion beam irradiation were energetic ions are focused to a target. The sputter yield can then be determined by weight loss measuring of the target probe after bombardment. However, at low bombarding energies - especially near the threshold energy of physical sputtering - ion beam intensities become very low. Therefore measured data at low impact energies are rare and more uncertain. Modeling can help to close this gap.

To calculate the physical sputtering yield in dependence on the impact energy and angle fit formulae have been developed. Mostly the Bohdansky formula and its revised version are used, which give the yield for normal incidence in dependent on the impact energy ${ }^{5}$. The overall accuracy of this formula is about a factor of $2-3$. Meanwhile several improvements of this analytic formula have been provided. New attempts have been done for a unified representation of the physical sputtering yield in dependent on the impact energy ${ }^{11}$. The dependence on the impact angle is described by the Yamamura formula ${ }^{12}$. Again the accuracy is not better than a factor of two.

A more detailed approach to calculate sputtering yields is based on the modeling of the transport of the impinging projectile inside the solid. The TRIM ${ }^{13}$ (TRansport of Ions in Matter) code and its derivative SDTrimSP ${ }^{14}$ follow the projectiles through a randomized target in the binary collision approximation (BCA) and calculate the collision cascade including recoils. The critical parameter is the potential describing the interaction between projectile and target atoms. Various potentials are in use, such as the screened Coulomb potential for $\mathrm{Kr}-\mathrm{C}^{15}$, which is a good approximation for many projectile-solid atom 
combinations. Within the BCA the interaction between the projectile and the target atoms is treated by successive two-body interactions. This approximation breaks down at low impact energies $(<\sim 10 \mathrm{eV})$ where many-body and quantum mechanical effects become important. More suitable for the situation of low impact energies $(<10 \mathrm{eV})$ are molecular dynamic (MD) simulations. Within MD calculations the motion is followed by the numerical solution of Newton's equations. For this, the many-body interaction potentials have to be known, which is a main constraint of MD calculations. Several methods exist to calculate these interaction potentials: the empirical approach ignores any quantum-mechanical effects or includes them by empirical methods. Semi-empirical potentials use the matrix representation from quantum mechanics, whereas the matrix elements themselves come from empirical formulae. Finally, ab-initio methods make use of full quantum-mechanical formulae. However, currently not all potentials necessary for plasma-wall interaction in fusion research are available - especially there is still a lack of data where beryllium is involved.

\section{A.3. Sputtering of layered systems and mixed materials}

So far only physical sputtering of pure elements has been described. The mixing of different materials caused by deposition or implantation of impurities at the solid, leads to additional processes. One example is the sputtering of a carbon layer on top of a tungsten substrate due to deuterium ion impact, a situation which can occur at the tungsten baffles in ITER.

Figure 5 shows the calculated (SDTrimSP) carbon sputtering yields in dependence on the deuterium ion impact energy for various thicknesses of a carbon layer on top of a tungsten substrate. As seen in figure 5, for thin carbon layers carbon sputtering becomes more effective compared with a pure carbon target. This can be explained by an increased reflection of incoming deuterium ions at the heavy tungsten substrate atoms compared to reflection on carbon atoms.

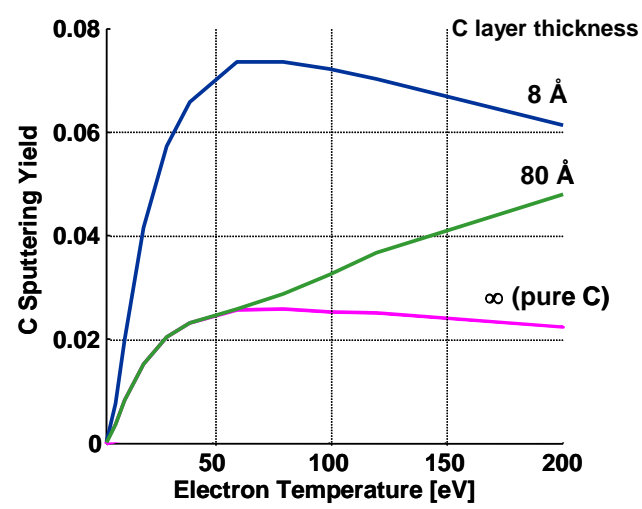

Figure 5: Physical sputtering yield of a carbon layer of varying thickness on top of a tungsten substrate (calculated with SDTrimSP). The impact energy $E_{\text {in }}$ of impinging $\mathrm{D}^{+}$ions is given as electron temperature $\mathrm{T}_{\mathrm{e}}\left(\mathrm{E}_{\text {in }} \sim 5 \mathrm{~T}_{\mathrm{e}}\right)$.
Thus, more of the penetrating deuterium ions are reflected back to the surface where sputtering of carbon takes place. The enhanced sputtering occurs especially for thin layers and high projectiles impact energies.

In a more realistic situation the particles are implanted with a certain depth profile leading to different concentrations, which also depends on exposure time. However, the basic processes influencing the sputter yield are the same but the effects can be less pronounced than shown in figure 5. In nearly all cases of multi-element systems preferential sputtering of one of the components occurs, which can be reproduced with the TRIM and SDTrimSP code ${ }^{16}$. Under multi-species conditions, further effects can occur like an oscillating of the partial sputtering yield in the case of heavy-ion bombardment of light targets (e.g. W ions on carbon target) ${ }^{17}$. This effect is explained with fluencedependent depth profiles of the implanted species. In addition to these collision-induced mechanisms, diffusion and segregation will influence the physical sputtering in mixed material systems.

\section{B. Chemical erosion and sputtering}

Chemical erosion involves thermal projectiles (in contrast to energetic ones in the process of chemical sputtering) initiating chemical reactions with surface atoms. In contrast to physical sputtering chemical erosion only occurs for specific combinations of projectile and target atoms. In fusion research chemical erosion of beryllium and carbon-based materials due to hydrogen (and its isotopes) are of main importance. Chemical erosion of carbon has been studied in great detail whereas the chemical erosion of beryllium is still subject of intense research.

Figure 6 summarizes the atomistic mechanisms leading to chemical erosion of carbon due to impact of thermal hydrogen atoms. Basic description of chemical erosion includes following processes: $\mathrm{C}$ atoms, bound in a $\mathrm{sp}^{2}$ configuration (bottom of figure 6 ) of the solid, are hydrogenised to $\mathrm{sp}^{3}$ complexes (top of figure 6) via an intermediate radical state $\mathrm{sp}^{\mathrm{x}}$ (left-hand side, figure 6).

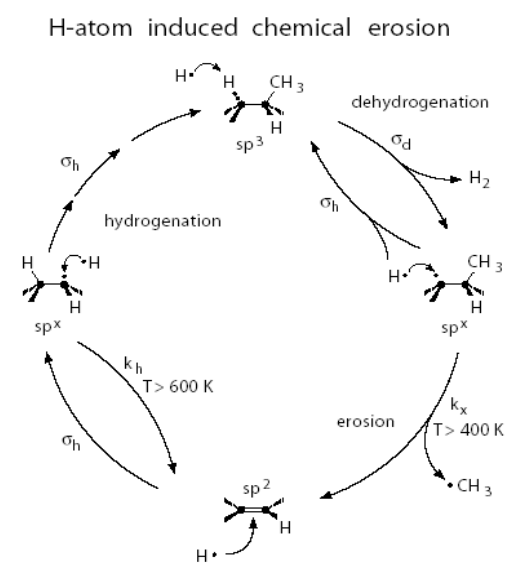

Figure 6: Atomistic processes involved in chemical erosion of carbon due to thermal hydrogen impact ${ }^{18}$. 
Further impinging hydrogen atoms will lead to formation of hydrogen molecules $\mathrm{H}_{2}$, which are desorbed and thus leaving a radical state $\mathrm{sp}^{\mathrm{x}}$ with a broken bond (right-hand side of figure 6). If the surface temperature is high enough (larger than $\sim 400 \mathrm{~K}$ ), chemical erosion can occur via desorption of hydrocarbon complexes. At higher surface temperatures (above about 600K) the intermediate radical state $\mathrm{sp}^{\mathrm{x}}$ can recombine with adsorbed atoms with a certain rate. This reduces the $\mathrm{sp}^{3}$ concentration and therefore leads to a decrease of chemical erosion. Altogether the chemical erosion can be described by the cross sections of hydrogenation $\sigma_{\mathrm{H}}$ and dehydrogenation $\sigma_{\mathrm{D}}$ and the surface temperaturedependant rate coefficients of desorption of hydrocarbon complexes $\mathrm{k}_{\mathrm{x}}$ and recombination of incoming $\mathrm{H}$ atoms with adsorbed ones $\mathrm{k}_{\mathrm{h}}$. The chemical erosion rate in steady state is given by the product of $\mathrm{k}_{\mathrm{x}}$ and the concentration $c_{s p^{x}}$ of $\mathrm{sp}^{\mathrm{x}}$ states, the latter one given as:

$$
c_{s p^{x}}=c_{s p^{3}} \frac{\sigma_{d} \Gamma_{H}}{\sigma_{h} \Gamma_{H}+k_{x}}
$$

with $\Gamma_{\mathrm{H}}$ as the impinging hydrogen atom flux. From this, the chemical erosion yield $\mathrm{Y}_{\text {therm, }}$, which is the erosion rate divided by the flux, follows to:

$$
Y_{\text {therm }}=\frac{c_{s p^{3}}}{\Gamma_{H}} \cdot \frac{\sigma_{d} \Gamma_{H}}{\sigma_{h} \Gamma_{H}+k_{x}} \cdot k_{x}
$$

In figure 7 , measured chemical erosion yields for bombardment of different carbon-based materials with deuterium or hydrogen atoms are presented in dependence on the surface temperature. In agreement with the above-described model the yield has a maximum at around $600 \mathrm{~K}$ and decreases with higher surface temperatures. In addition, the measurements show a strong dependence on the carbon material.

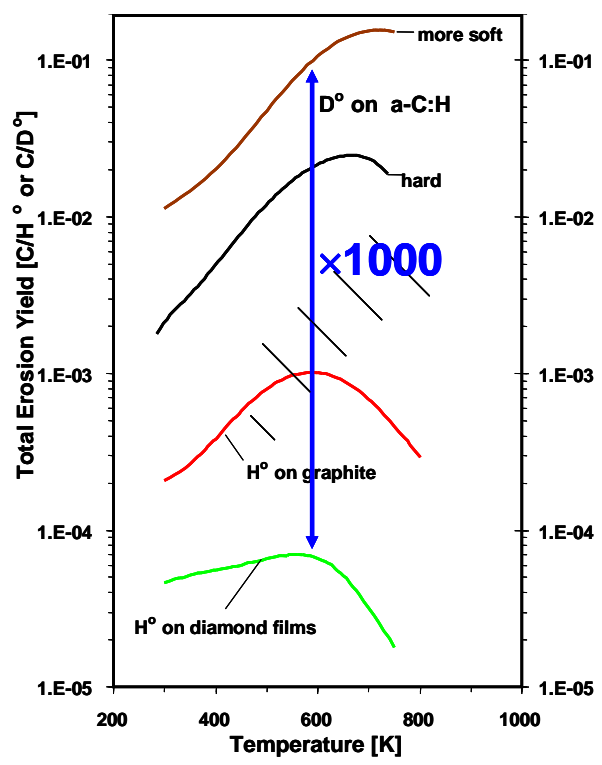

Figure 7: Chemical erosion yield for bombardment of different carbon-based materials with thermal hydrogen/ deuterium atoms ${ }^{19}$.
Amorphous a-C:H carbon films (in the figure marked as "soft" and "hard") suffer from much larger chemical erosion than graphite or pure diamond films. This can be explained in the model with the concentration of $\mathrm{sp}^{\mathrm{x}}$ states, which strongly depends on the material structure.

A wide range of hydrocarbon species can be formed chemically. With thermal hydrogen atom impact $\mathrm{CH}_{3}$ is formed, while $\mathrm{CH}_{4}$ dominates at higher ion impact energies. In addition, a large family of higher hydrocarbons $\mathrm{C}_{2} \mathrm{H}_{\mathrm{X}}$ and $\mathrm{C}_{3} \mathrm{H}_{\mathrm{X}}$ is observed. Normally the energy spectrum of eroded species can be described with a Maxwell distribution around the surface temperature:

$$
N\left(E_{\text {therm }}\right) \propto E_{\text {therm }} \cdot e^{-E_{\text {therm }} / k T_{\text {surf }}}
$$

Similar to physical sputtering the angular distribution can be approximated with a cosine function.

Chemical sputtering is defined as process where due to ion bombardment a chemical reaction occurs, which produces a particle weakly bound to the surface which then can be desorbed into the gas phase. The ion bombardment promotes the chemical reaction whereas the release of the particle itself is mainly thermally driven. Chemical sputtering depends on the kinetic energy and the chemical reactivity of the impinging species. The eroded species are molecules formed out of projectile and target atoms. In contrast to physical sputtering but similar to chemical erosion, chemical sputtering occurs only for certain combinations of projectile and target material. The following discussion will focus on the chemical sputtering of carbon-based materials. The threshold energy for chemical sputtering is significantly smaller than for physical sputtering and the chemical sputtering yield shows a clear dependence on the surface temperature of the substrate. As for chemical erosion also chemical sputtering leads to a wide range of sputtered hydrocarbon species. In addition to the surface temperature dependence the distribution of sputtered species also depends on the ion impact energy.

The energetic hydrogen ions penetrate into the solid and as long as they have enough energy the interaction with the solid atoms is determined by collision effects (leading to displacement of target atoms or physical sputtering). At the end of the projectile's trajectory, after thermalisation, chemical effects become important. This can be described by the model of chemical erosion as presented in the previous chapter - a hydrocarbon complex can be formed with a yield $\mathrm{Y}_{\text {therm. }}$. The hydrocarbon at the end of the ion range can then diffuse to the surface where it finally can leave the solid. However, in case of chemical sputtering the yield is enhanced compared to chemical erosion due to the effect of radiation damage of the penetrating energetic ion. The radiation damage in form of broken $\mathrm{C}-\mathrm{C}$ bonds provides additional reaction sites for incoming $\mathrm{H}$ atoms and thus increases the probability of hydrocarbon formation. The yield for the enhanced thermal reaction can be written as

$$
Y_{\text {therm }}^{\text {damage }}=Y_{\text {therm }} \cdot\left(1+D \cdot Y_{\text {dam }}\right)
$$


Here $Y_{d a m}$ is the radiation damage yield, $D$ a fit parameter to match experimental results. For $\mathrm{Y}_{\mathrm{dam}}$ one usually uses the physical sputtering yield but with a lower threshold energy. In addition to this damageinduced effect a process at the surface comes into play. It is observed experimentally that the hydrocarbon release under energetic ion bombardment starts at smaller surface temperatures than with thermal atom bombardment. This is explained by means of physical sputtering of weakly bound $\mathrm{sp}^{3} \mathrm{CH}_{\mathrm{x}}$ groups from the surface and described with a yield $\mathrm{Y}_{\text {surf }}$. The chemical sputtering yield can then be written as

$$
Y_{\text {chem }}^{\text {sputter }}=Y_{\text {therm }} \cdot\left(1+D \cdot Y_{\text {dam }}\right)+Y_{\text {surf }}
$$

The yield according to Eq. (11) depends on surface temperature, energy and flux of impinging hydrogen ions. By means of comparison with experimental data this has been used to formulate a semi-empirical formula to describe theses dependencies in detail ${ }^{20}$.

\section{Energy dependence of $Y_{\text {chem }}^{\text {sputer }}$}

The chemical sputtering yield calculated according the formula in ${ }^{20}$ is plotted in figure 8 in dependence on the impact energy for two surface temperatures and a hydrogen flux of $1 \cdot 10^{22} \mathrm{~m}^{-2} \mathrm{~s}^{-1}$. At energies below $\sim 2 \mathrm{eV}$ only the thermal erosion process is active. At higher impact energies the yield is determined by the damageinduced $\left(\mathrm{Y}_{\mathrm{dam}}\right)$ and the surface erosion $\left(\mathrm{Y}_{\text {surf }}\right)$ effect. The qualitative energy dependence is therefore similar to the one of physical sputtering (see figure 2).

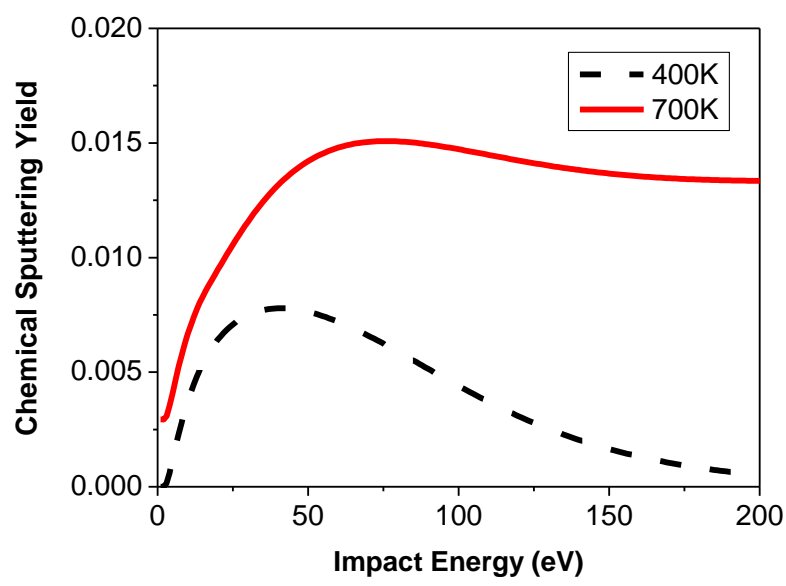

Figure 8: Calculated chemical sputtering yield in dependence on hydrogen impact energy for surface temperatures of 400 and $700 \mathrm{~K}$ ( for $\Gamma_{\mathrm{H}}=1 \cdot 10^{22} \mathrm{~m}^{-2} \mathrm{~s}^{-1}$ ).

\section{Surface temperature dependence of $Y_{\text {chem }}^{\text {sputter }}$}

Similar to chemical erosion also chemical sputtering shows a dependence on surface temperature. The common observation is a maximum of the sputtering yield about 900K. However, as will be discussed next, the surface temperature at which this maximum occurs also depends on the impinging hydrogen flux.

\section{Flux dependence of $Y_{\text {chem }}^{\text {sputer }}$}

A compilation of data from various experiments (ion beam devices, linear plasma machines as well as tokamaks) indicates a strong flux dependence of the chemical sputtering yield: with increasing incoming hydrogen flux the yield decreases. Figure 9 shows experimental data together with the graph according to the semi-empirical formula (black line) for chemical sputtering. For comparison the experimental data are normalized to an impact energy of $30 \mathrm{eV}$ and the surface temperature of maximum yield. The flux dependence of the chemical sputtering yield can be understood in terms of the thermal reaction cycle. This predicts an increase of the temperature, where the maximum of chemical sputtering occurs, with flux. At these high surface temperatures the thermodynamic equilibrium of the H/C system shifts from hydrocarbon formation to $\mathrm{H}_{2}$ release. Therefore, the chemical sputtering yield decreases with increasing flux.

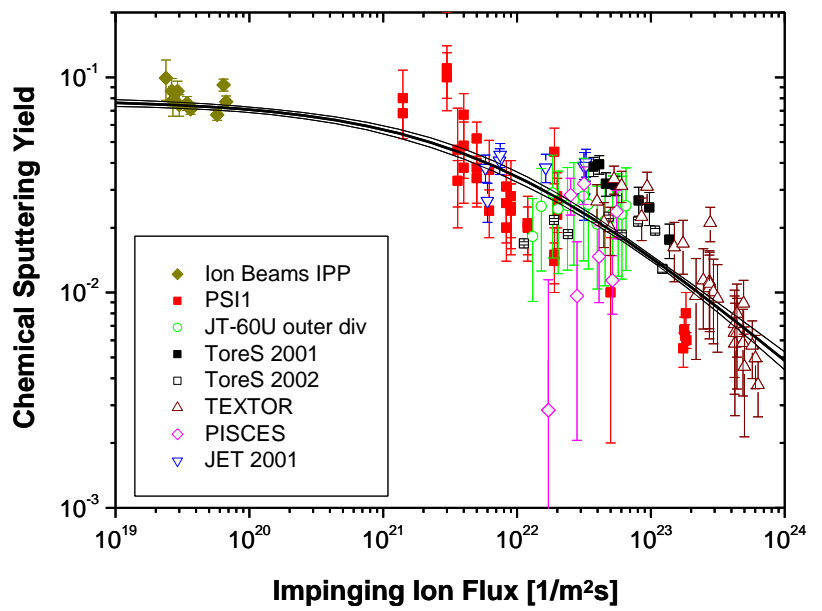

Figure 9: Chemical sputtering yield in dependence on impinging hydrogen ion flux ${ }^{21}$.

\section{Synergistic effects}

Simultaneous bombardment of a carbon surface with thermal hydrogen and energetic ions (e.g. Ar) shows an enhanced carbon erosion compared to bombardment with hydrogen atoms only ${ }^{22}$. This can be explained with the above-described model of chemical sputtering: the energetic ions produce broken bonds, which serve as reaction sites for the impinging hydrogen atoms. In addition, the energetic ions can sputter hydrocarbon complexes from the surface.

Also pre-irradiated graphite surfaces suffer from larger carbon erosion than untreated surfaces ${ }^{23}$. Again, the ions produce dangling bonds during the preirradiation procedure, which then lead to an increased chemical sputtering and erosion. 


\section{Other erosion mechanisms}

\section{C.1. Blistering}

In laboratory experiments it is seen that high fluences (the fluence is the time-integrated flux) of light ions, such as hydrogen and its isotopes or helium, can cause blistering on metal surfaces like tungsten ${ }^{24}$. This process is caused by trapping of gas atoms inside bubbles at the surface of the metal, which leads to very high pressures inside the bubble. Blistering can lead to enhanced erosion due to flaking of surface material, grain ejection or evaporation of thin blister caps. For helium impact on tungsten the critical fluence at which blistering starts is about $10^{21}$ to $10^{22} \mathrm{He}$ atoms $/ \mathrm{m}^{2}$. In case of $\mathrm{H}$ isotopes it is about two orders of magnitude higher - on tungsten blistering starts at about $10^{24} \mathrm{D} / \mathrm{m}^{2}$. The surface temperature range for $\mathrm{H}$ blistering on tungsten is $<600^{\circ} \mathrm{C}$ whereas it goes to higher temperatures for helium.

The influence on blistering of carbon impurity impinging on a tungsten surface has been investigated in 25 . It is seen that hydrogen blistering occurs at a target temperature of $650 \mathrm{~K}$ and a carbon concentration of $0.95 \%$ whereas with lower carbon concentrations $(0.11 \%)$ or higher surface temperatures no significant blistering is found. One possible explanation could be the formation of a carbide layer at top of the surface, which enhances hydrogen diffusion beyond the ion range and the carbide layer into the bulk (the solubility of hydrogen in WC is low). Then voids could be created in the bulk, which can develop to blisters. The decrease of blistering at higher surface temperatures could result from the higher thermal energy of hydrogen at which traps triggering the blistering are not active anymore.

Whereas in existing fusion experiments significant blistering has not yet been observed this could be different in ITER and next-step fusion machines. Especially the effect of alpha particles $\left(\mathrm{He}^{+}\right)$- which are a product of fusion reactions - has to be taken into account.

\section{C.2. Radiation enhanced sublimation (RES)}

In case of carbon-based materials anomalously enhanced erosion has been observed at elevated surface temperatures in laboratory experiments ${ }^{26}$. Figure 10 shows the total erosion yield as result of argon ion bombardment $(5 \mathrm{keV})$ on graphite in dependence on the surface temperature. Whereas the erosion yield is constant up to about $1000 \mathrm{~K}$ and can be explained with physical sputtering it increases with higher surface temperatures. The increase starts clearly below the sublimation temperature of graphite (about 3200K) and can be described with an exponential function:

$$
Y=Y_{0} \cdot \exp \left(-E_{R E S} / k T\right)
$$

In Eq. (12) $E_{R E S}$ is the activation energy for radiation enhanced sublimation $(0.6-0.9 \mathrm{eV})$ and $\mathrm{Y}_{0}$ a pre-factor. RES is explained by the production of radiation defects (interstitials and vacancies) due to the energetic ions. The diffusion of the interstitials to the surface then competes with the annihilation with vacancies. Interstitials, which survive annihilation with vacancies, can arrive at the surface and desorb into the gas phase. However, up to now RES has not been observed clearly under tokamak particle impact conditions as e.g. shown in ${ }^{27}$. This might be due to the high fluxes in combination with low energies.

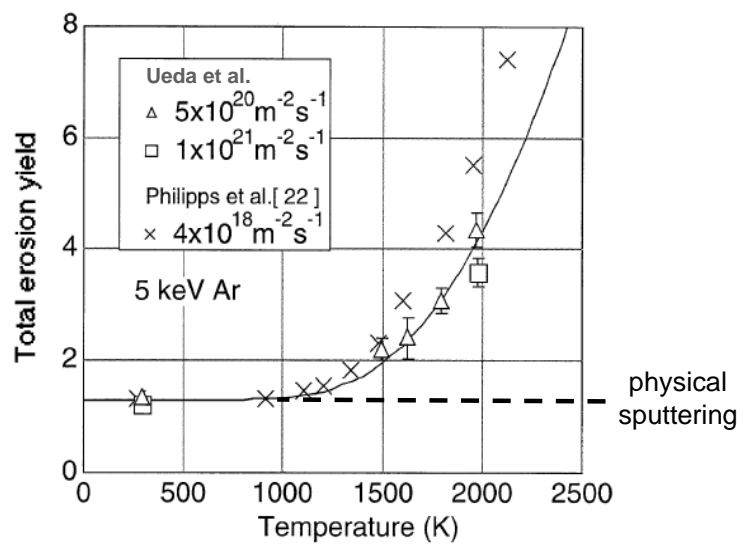

Figure 10: Total erosion yield of graphite due to $\mathrm{Ar}^{+}$ ion bombardment in dependence on surface temperature ${ }^{26}$.

\section{DEPOSITION MECHANISMS}

\section{A. Reflection \& deposition}

A projectile hitting a surface can be reflected (backscattered) from the surface with a certain probability which is expressed by the reflection coefficient $\mathrm{R}(0 \leq \mathrm{R} \leq 1)$. Thus, the probability for a projectile of being deposited is $1-\mathrm{R}$. The reflected particles are in most cases neutrals. Similar to erosion yields reflection coefficients of atoms can be measured under well-defined conditions in ion beam experiments. In case of molecular species other methods are in use as discussed later. Reflection data at fusion relevant low impact energies are rare. For calculation of reflection coefficients the same tools used for sputtering yield calculations can be applied (BCA based calculations such as TRIM, or MD simulations).

\section{A.1. Atomic species}

At first it is assumed that the projectile atoms interact with a smooth surface. Since reflection is governed by collisions between projectile and target atoms, the reflection coefficient depends on projectile and target masses $\left(\mathbf{M}_{1}, \mathbf{M}_{2}\right)$ and impact energy and angle $\left(\mathrm{E}_{0}, \alpha_{0}\right)$. Generally, the reflection coefficient increases with increasing mass ratio $\mathrm{M}_{2} / \mathrm{M}_{1}$ - the reflection of light projectiles at heavy substrate atoms is very effective.

As example, the energy dependence of the reflection coefficient for carbon on carbon at an impact angle of $45^{\circ}$ calculated with TRIM is shown in figure 11 . 


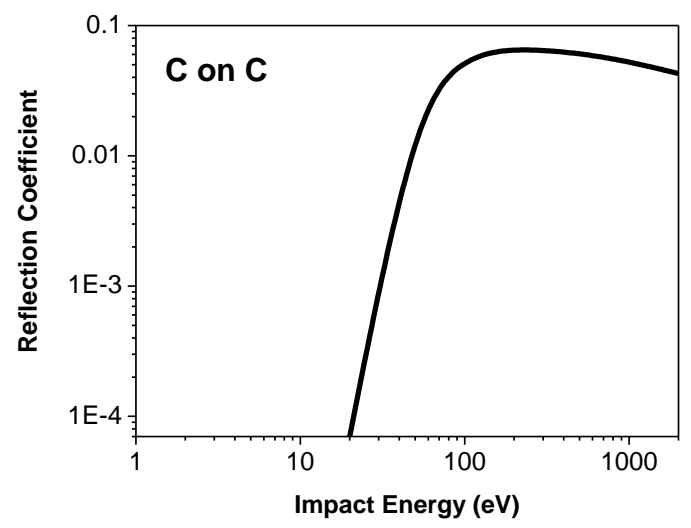

Figure 11: Energy dependence of the reflection coefficient $\mathrm{R}$ for carbon on carbon at impact angle of $45^{\circ}$ (TRIM).

At impact energies larger than $\sim 200 \mathrm{eV}$ the reflection coefficient decreases monotonically - the projectiles penetrate deeper into the solid and the probability of implantation increases. The TRIM calculations show a steep decrease of reflection going to smaller impact energies. At $\mathrm{E}_{0}<20 \mathrm{eV}$ the calculated reflection coefficient (figure 11) equals zero. However, as discussed in the previous chapter, the BCA method is not valid at such small impact energies below about 10 eV. MD calculations must be used under those conditions showing in contrast non-zero reflection coefficients even at impact energies less than $10 \mathrm{eV}^{28,29}$.

The dependence of reflection on the impact angle is presented in figure 12 based on TRIM calculations for $\mathrm{C}$ on $\mathrm{C}$ at impact energy of $200 \mathrm{eV}$. With increasing angle of incidence the reflection probability increases: with more grazing incidence the projectile penetrates less deep into the solid which decreases the implantation probability.

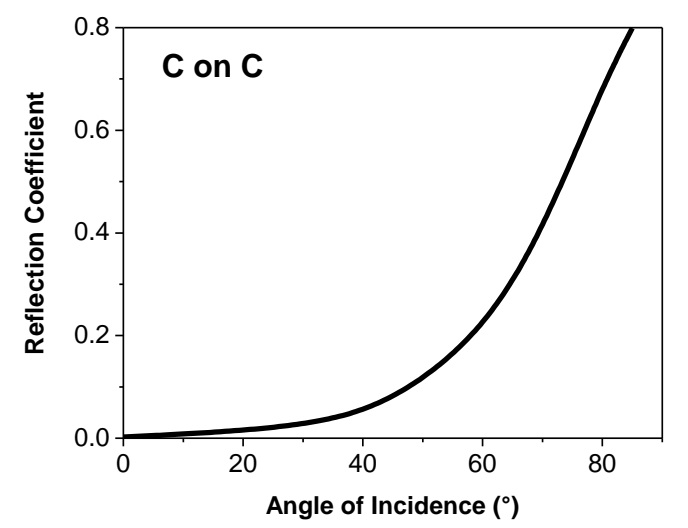

Figure 12: Angle dependence of reflection coefficient $\mathrm{R}$ for carbon on carbon at impact energy of $200 \mathrm{eV}$ (TRIM).

The energy distribution of reflected particles depends on projectile - solid combination, impact energy and angle of projectile. For Maxwell-distributed projectiles the energy distribution of reflected particles can be described with an exponential decrease ${ }^{30}$.
Significant deviations from this occur only for impact energies smaller than $200 \mathrm{eV}$.

At energies not too large (reduced energy $\varepsilon<\sim 10$, where $\varepsilon=\frac{M_{1}}{M_{1}+M_{2}} \cdot \frac{a_{S}}{Z_{1} Z_{2} e^{2}} \cdot E_{0}$ with $a_{S}$ the screening length and e the electron charge) and normal incidence the reflected particles have a cosine distribution, but deviations occur for different conditions. Nevertheless, for isotropic bombardment a cosine distribution is still a good approximation.

\section{Reflection at rough surfaces}

As discussed in the previous chapter II.A. surface roughness will change the local angle of incidence of projectiles compared to the nominal one. In ${ }^{31}$ the case of carbon bombardment onto a rough tungsten surface is discussed. For a nominal angle of incidence of $0^{\circ}$ the carbon reflection is increased compared to a smooth surface. The measured reflection on the rough surface can be explained with a mean local angle of incidence of $38^{\circ}$ instead of $0^{\circ}$. Similar results are obtained for a nominal angle of incidence of $60^{\circ}$ (mean local angle of about $70^{\circ}$ ).

\section{Prompt deposition}

In fusion experiments magnetic fields are applied to ensure confinement ${ }^{32}$. Eroded and sputtered particles normally start as neutrals from the surface but are ionized at some distance (ionization length $\lambda_{\text {ion }}$ ) depending on the local plasma parameters. The magnetic field then leads to a gyration movement of the charged particle with a certain Larmor radius $r_{L}$. As can be seen from figure 13 , there is some probability for the particle to return to the surface (where it then can be deposited with a probability of 1-R) within the first gyration if the Larmor radius is larger than the ionization length.

\section{$\otimes B$}

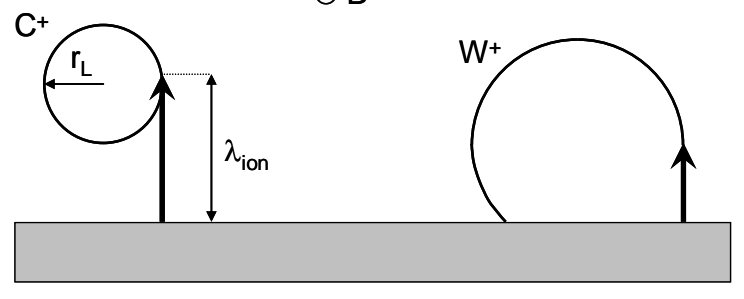

Figure 13: Schematic view of prompt deposition for tungsten $\mathrm{W}^{+}$ions.

From this a criterion for prompt deposition can be derived based on the following formulae:

$$
\lambda_{\text {ion }}=\frac{v_{\perp}}{\langle\sigma v\rangle_{\text {ion }} \cdot n_{e}}
$$

where $\langle\sigma v\rangle_{i o n}$ in $[\mathrm{m} 3 / \mathrm{s}]$ is the ionization probability,

$$
\begin{gathered}
r_{L}=\frac{M \cdot v_{\perp}}{Q \cdot B} \\
P_{\text {prompt }}=\frac{\lambda_{\text {ion }}}{r_{L}}=\frac{B}{\langle\sigma v\rangle_{\text {ion }} \cdot n_{e} \cdot M} \quad \text { for } Q=1
\end{gathered}
$$


If $\mathrm{P}_{\text {prompt }}<1$ prompt deposition becomes possible. From Eq. (13c) follows that prompt deposition especially occurs for high-Z materials of high mass $\mathrm{M}$ and in case of large ionization probability $\langle\sigma v\rangle_{\text {ion. }}$. This is also illustrated schematically in figure 13 for tungsten (high$\mathrm{Z})$ in comparison to carbon (low-Z).

\section{A.2. Sticking of hydrocarbons}

As discussed in chapter II., chemical erosion/ sputtering of graphite walls leads to the formation of hydrocarbons $\mathrm{C}_{\mathrm{x}} \mathrm{H}_{\mathrm{y}}$ (here $\mathrm{H}$ represents hydrogen and its isotopes $\mathrm{D}$ and $\mathrm{T}$ ), which are released into the plasma. These species can also return to wall elements and stick to the surface and form hydrocarbon layers. Direct measurements of sticking coefficients of hydrocarbons are rare since quantified radical sources for the species of interest are needed, which requires significant experimental efforts. As alternative to the sticking coefficient the surface loss probability can be measured by means of the cavity technique ${ }^{33}$, which is more practicable. The surface loss probability $\beta$ of a hydrocarbon equals the sum of the sticking probability $S$ and the probability $\gamma$ of the hydrocarbon to react to a non-reactive volatile product via surface reactions. The surface loss probability is thus an upper limiter for the sticking probability.

$$
\beta=\mathrm{S}+\gamma, \text { with } \mathrm{S}+\gamma+\mathrm{R}=1
$$

The cavity technique uses a closed volume with a small entrance slit and hydrocarbons entering this cavity will lead to deposition on the inside walls. With the measured deposition profiles and applying a transport model for hydrocarbons inside the cavity, the surface loss probabilities for the various species are obtained. It is seen that the surface loss probability significantly depends on the hybridization of the radical: $\beta\left(\mathrm{sp}^{1}\right) \sim 0.8$, $\beta\left(\mathrm{sp}^{2}\right) \sim 0.35$ and $\beta\left(\mathrm{sp}^{3}\right) \sim 10^{-3}$. Therefore, especially unsaturated hydrocarbons contribute to film growth. These $\beta$ values have been obtained with the cavity surface at room temperature. At higher surface temperatures erosion effects become important such that the surface loss probability can become negative (at $\mathrm{T}_{\text {surf }}$ around $600 \mathrm{~K})^{34}$. At even higher surface temperatures (> $700 \mathrm{~K})$ graphitization can take place, which then results in positive loss probabilities associated with film growth. Further experimental data on surface loss probabilities can be found in ${ }^{35}$.

Molecular dynamics modeling can be applied to calculate sticking coefficients (or surface loss probabilities) for hydrocarbon species. Compared to the experiments, modeling can more easily study the influence of incident energy, angle and surface conditions. As example, figure 14 shows modeled and measured data for $\mathrm{CH}_{2}$ and $\mathrm{CH}_{3}{ }^{36}$. The experimental data, taken from ${ }^{37,38}$, are obtained at thermal energies for incoming species. The films were growing under direct plasma contact. Therefore it can be assumed that hard, saturated graphite films did develop.

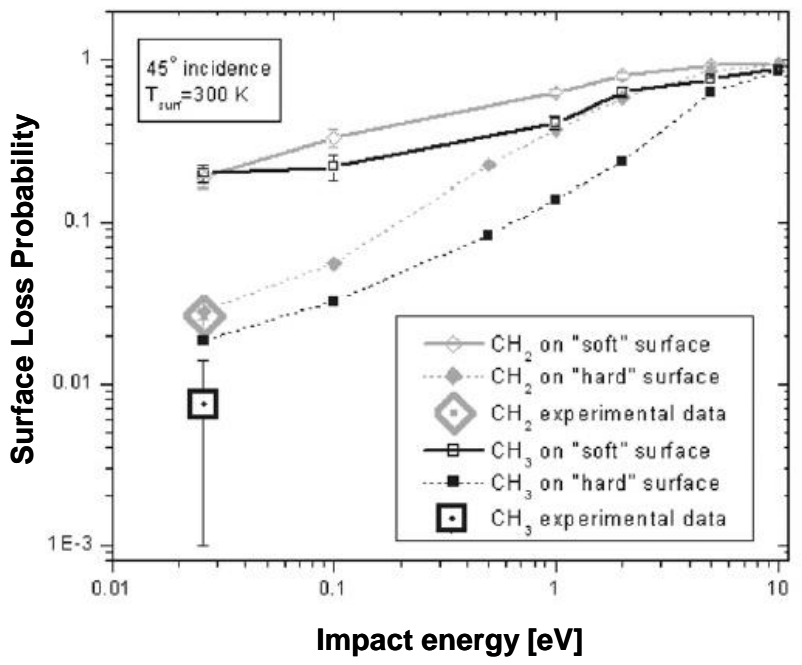

Figure 14: Measured and modeled (Molecular Dynamics) surface loss probabilities for $\mathrm{CH}_{2}$ and $\mathrm{CH}_{3}{ }^{36}$.

As can be seen in figure 14, only the assumption of a hard surface results in a fair agreement between modeled and measured value of the surface loss probability.

More molecular dynamic modeling results of surface loss probabilities can be found e.g. in ${ }^{39,40}$.

\section{B. Adsorption}

Up to now the deposition has been discussed by implantation of energetic particles into a solid or layer formation on top of it. The impinging particle is thermalized either inside the solid at a certain depth where it forms a binding with the solid atoms at the location where it comes to rest or in the near surface layer of a growing deposition film. Apart from these processes, thermal particles can also be adsorbed at the solid surface. Especially gaseous species (like $\mathrm{O}_{2}$ or $\mathrm{H}_{2}$ ) can form adsorbat layers. Adsorption is possible because the surface atoms of a solid have unsaturated bindings. Therefore it is energetically beneficial to form bindings with other atoms or molecules. Adsorption can be realized via two mechanisms: in case of physisorption the binding between the adsorbat and the solid surface atom is realized via van der Waals forces - which involve no change of the chemical structure of adsorbat and solid surface atom. The binding energy through van der Waals forces is less than about $0.5 \mathrm{eV}$. In case of chemisorption the binding between adsorbat and solid surface atom happens through the exchange or sharing of electrons resulting in binding energies of about several $\mathrm{eV}$. The rate of adsorption depends on the material combination, the surface structure and temperature. Adsorbed species can be released from the surface via thermal desorption, ion induced collisions and also photons. With increasing surface temperature the rate of desorption increases. In fusion experiments the ioninduced desorption is the most important desorption process. 


\section{Mechanisms of fuel retention}

Retention of the radioactive fuel tritium in the walls of fusion devices is a major concern for future fusion devices since the in-vessel amount of tritium is limited from safety aspects. If a certain limit is reached, plasma operation has to be stopped and the wall has to be cleaned. This limits the availability of the device and demonstrates the need to develop effective cleaning methods, which presently are only marginally developed.

\section{Adsorption}

Tritium can be adsorbed at the surface. This mechanism saturates - e.g. in case of a carbon when the surfaces of open porosity are filled. Due to the weak bonding between the adsorbed fuel and the surface atoms this retention mechanism is transient.

\section{Implantation}

Energetic tritium particles are trapped by chemical bonding in the material at a certain depth where they come to rest. This mechanism is permanent because of the strong binding between $\mathrm{T}$ and the solid atoms. However, it saturates when the maximum possible tritium density is reached.

\section{Bulk diffusion}

At higher surface temperatures diffusion into the bulk becomes important. This mechanism is permanent and does not saturate but depends on the diffusion coefficient and also on the density of traps at which the diffusing $\mathrm{T}$ can be bound quasi permanently. This process can become important for high $\mathrm{Z}$ plasma facing materials (e.g. W) under long-pulse operation and under high fluxes as in ITER and next step devices.

\section{Co-deposition}

Eroded material will be deposited somewhere and can thus lead to formation of layers if the deposition does not occur on the location of the material erosion. These layers will contain a certain amount of tritium due to co-deposition with the wall material. The tritium content of co-deposited layers shows a complicated parameter dependence on the layer composition, layer microstructure (density or porosity) and surface temperature. Tritium retention due to co-deposition is permanent and not saturating. This mechanism dominates the overall long-term tritium retention in devices with low-Z walls, which have comparably large erosion rates. If deposited layers become instable, flaking can occur and leading to dust formation inside the device.

\section{Transmutation}

In addition, neutrons (as result from fusion reactions) impinging on a beryllium surface will lead to the production of tritium via nuclear reactions, called neutron transmutation. The energetic neutrons produce also damages inside the wall materials, which then can serve as trapping sites for tritium and therefore increase the tritium retention.

\section{EROSION AND DEPOSITION IN FUSION EXPERIMENTS}

Wall elements in fusion experiments have contact with the edge plasma and therefore a certain plasma ion flux will hit the wall. An edge plasma with electron temperature $T_{e}$, ion temperature $T_{i}$ and electron density $\mathrm{n}_{\mathrm{e}}$ leads in case of a hydrogen plasma to an hydrogen ion flux $\Gamma_{\mathrm{H}^{+}}$of

$$
\Gamma_{H^{+}}=c_{S} \cdot n_{e} \text { with } \quad c_{S}=\sqrt{\frac{T_{e}+T_{i}}{M_{H}}}
$$

where $c_{S}$ is the acoustic sound speed and $M_{H}$ the mass of hydrogen atoms. In addition to plasma ions also impurity ions strike the wall elements. According to the wall materials in use in present experiments these are mainly carbon, tungsten and beryllium. Besides this, there is always a certain oxygen impurity influx. Depending on the experimental conditions additional impurity fluxes can be important such as argon or neon, which are injected into the edge plasma for cooling issues. The local plasma parameters also define the amount of neutrals hitting the wall. Finally, in case of a fusion experiment with a significant amount of fusion reactions also helium ions and neutrons will hit the surrounding walls. Obviously, compared to a laboratory experiment the situation in a tokamak is much more complex: instead of one projectile species there is a whole bunch of impinging projectiles, which in addition are not mono-energetic but have a certain energy distribution.

In the following some selected examples of erosion, transport and deposition experiments will be described. Possible implications for future fusion experiments, especially ITER, will be discussed. Methods of erosion and deposition measurements are described in ${ }^{41}$.

\section{A. Erosion and deposition experiments in TEXTOR}

TEXTOR (Torus EXperiment for Technology Oriented Research, sited in Jülich, Germany) is a medium size limiter tokamak with a large plasma radius of $1.75 \mathrm{~m}$ and a small plasma radius of $0.48 \mathrm{~m}^{42}$. The limiter configuration of tokamaks is described in ${ }^{32}$. TEXTOR is an overall carbon machine. It is equipped with two limiter locks, which enable well diagnosed experiments under wel- defined plasma conditions.

\section{A.1. Measurement of chemical sputtering in TEXTOR}

A spherically shaped graphite test limiter is exposed to the edge plasma of TEXTOR, which has been heated externally to study the dependence of chemical sputtering on surface temperature in detail. The chemical sputtering yield is measured by observing the CD emission near the limiter surface, which is a dissociation decay product of methane $\mathrm{CD}_{4}$, which itself is chemically sputtered. To obtain the eroded $\mathrm{CD}_{4}$ flux from the measured $\mathrm{CD}$ light one needs the so-called $\mathrm{D} / \mathrm{XB}$ value, which is the ratio of $\mathrm{CD}_{4}$ particles and corresponding $\mathrm{CD}$ emission. $\mathrm{D} / \mathrm{XB}$ values have to be determined independently. The best procedure is to 
inject under the same conditions a defined amount of $\mathrm{CD}_{4}$ and measuring the resulting $\mathrm{CD}$ emission. Figure 15 presents methane formation yields from test limiters in TEXTOR at a deuterium flux of about $2 \cdot 10^{22} \mathrm{~m}^{-2} \mathrm{~s}^{-1}$. More details of this experiment can be found in ${ }^{43}$. The surface temperature dependence of chemical sputtering yield corresponds well with the one described in chapter II. After a maximum yield at a certain temperature a significant decrease arises at higher temperatures. Maximum yield of about $4 \%$ occurs at a surface temperature of $\sim 950 \mathrm{~K}$.

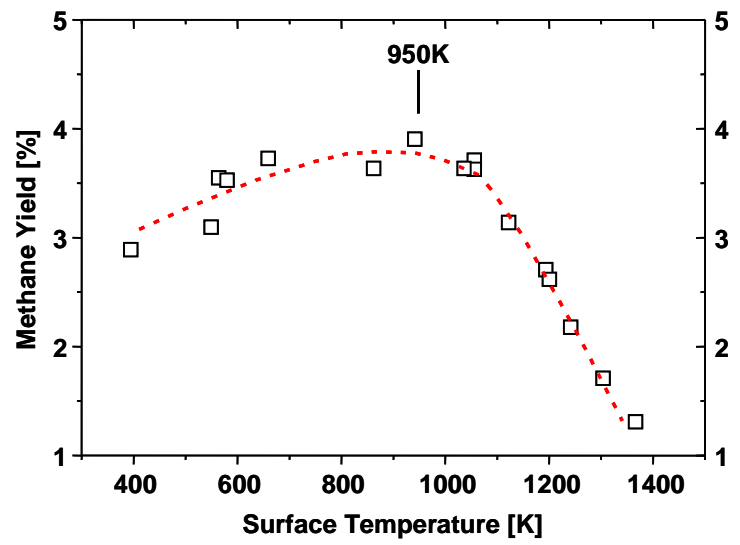

Figure 15: Methane formation yield (left y-axis) in dependent on surface temperature measured at a graphite test limiter exposed to the edge plasma of TEXTOR $^{43}$.

\section{A.2. Methane injection experiments in TEXTOR}

Deposition of impurities is an important issue for ITER, mainly due to tritium retention by co-deposition in such layers. In TEXTOR this has been investigated in detail by injecting ${ }^{13} \mathrm{C}$ marked methane $\mathrm{CH}_{4}$ through test limiters of different shape (spherical or roof-like) and material (graphite, tungsten and molybdenum) ${ }^{44,45}$. The ${ }^{13} \mathrm{C}$ marked methane has been chosen to distinguish natural ${ }^{12} \mathrm{C}$ deposition caused by background plasma from the deposition caused by local injection. Figure 16 shows exemplarily the tungsten limiters (roof-like and spherical shape) after local ${ }^{13} \mathrm{CH}_{4}$ injection demonstrating deposition near the injection hole. The broader dark region on the bottom part of the spherical limiter results from carbon ${ }^{12} \mathrm{C}$ deposition.
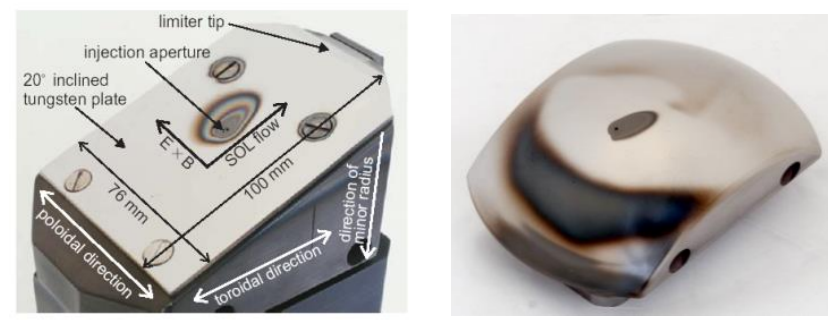

Figure 16: Tungsten test limiters after plasma exposure in TEXTOR with local methane ${ }^{13} \mathrm{CH}_{4}$ injection.
In all these experiments the local ${ }^{13} \mathrm{C}$ deposition efficiency (ratio of locally deposited ${ }^{13} \mathrm{C}$ on the test limiter surface and injected ${ }^{13} \mathrm{C}$ ) was very small: for spherical limiters $0.3 \%$ on tungsten and $4 \%$ on graphite and for roof-like limiters $0.17 \%$ on molybdenum, $0.11 \%$ on tungsten and $0.17 \%$ on graphite. The substrate dependence of the ${ }^{13} \mathrm{C}$ deposition can be reproduced by SDTrimSP and is explained by a more effective erosion of thin carbon layers if the underlying substrate has a higher atom mass, see also figure 5 . The larger deposition efficiency on spherical limiters can be explained with a flux dilution due to grazing incidence of the magnetic field at top of these limiters, leading to decreased erosion of deposited material.

The described experiments have been modeled with the impurity transport code $\mathrm{ERO}{ }^{46}$. The low ${ }^{13} \mathrm{C}$ deposition efficiencies can be reproduced only if an enhanced erosion of deposited carbon compared to graphite at plasma-wetted areas is assumed ${ }^{47,48}$. Using "standard" values for hydrocarbon sticking and reerosion of deposited carbon, the modeled ${ }^{13} \mathrm{C}$ deposition efficiency is typically in the $50 \%$ range. A possible explanation for this enhanced erosion is an ion-induced desorption of loosely bound hydrocarbons that are freshly deposited on the surface. It has to be noted that also from injection experiments with $\mathrm{WF}_{6}$ and $\mathrm{SiH}_{4}$ similar conclusions have been drawn concerning the insitu enhanced erosion of deposits. Thus, this effect can have direct influence on ITER since it provides a process for impurity transport at plasma-wetted areas triggered by successive re-deposition and re-erosion until finally layer formation (and tritium retention) takes place at plasma-shadowed regions.

Experiments with varying surface roughness show an increase of ${ }^{13} \mathrm{C}$ deposition with roughness. Particles deposited inside the valleys of a rough surface are obviously more protected from the incident flux, which in the overall decreases the erosion of deposited ${ }^{13} \mathrm{C}$. This is in agreement with the effect of surface roughness on physical sputtering as discussed in chapter II.A.

\section{A.3. High-Z test limiter experiments in TEXTOR}

The sputtering of high- $Z$ materials has been investigated on test limiters by in-situ by spectroscopy. It is seen that the effective sputtering yield normalized to the impinging deuterium ion flux varies between $0.5 \%$ at high edge density and $3 \%$ at low density ${ }^{49}$. These yields cannot arise from deuterium sputtering alone but actually are dominated by carbon and oxygen impurity sputtering. Comparison with calculated sputtering yields lead to good agreement if also prompt deposition of sputtered tungsten is taken into account. The erosion of tungsten from these limiters at elevated surface temperatures up to melting of $\mathrm{W}(3700 \mathrm{~K})$ does not show an enhanced yield compared to the expected physical sputtering ${ }^{50}$. 


\section{B. ELM-induced enhanced erosion in JET}

JET (Joint European Torus, located in Culham, UK) is presently the largest fusion research experiment in the world and therefore the most ITER-relevant device with respect to size and magnetic field configuration. The major plasma radius is $2.96 \mathrm{~m}$ and the minor radius of the D-shaped plasma is $2.1 \mathrm{~m}$ in vertical and $1.25 \mathrm{~m}$ in horizontal direction. As ITER, it is a divertor machine, in which the magnetic field lines are diverted by means of special coils into the divertor chamber. At the divertor plates the main plasma-wall interaction takes place. Details of the divertor concept can be found in ${ }^{32}$. Main wall and divertor tiles of JET are made out of graphite.

Deposition in the divertor of JET can be measured shot-resolved with Quartz Micro Balances (QMB) ${ }^{41}$. One QMB is mounted in the inner divertor of JET (see figure 17.). With the magnetic configuration as indicated in figure 17, deposition at this QMB represents erosion on tile \#4, where the strike point (SP) is located. The right part of figure 17 shows the carbon deposition on the QMB for high confinement discharges (H-mode) in dependent on ELM energy to the divertor - ELMs are periodic energy bursts typical for $\mathrm{H}$-mode discharges and are seen as danger for ITER. The observed carbon deposition on the QMB (and thus erosion at the SP) in dependent on ELM energy cannot be explained with physical sputtering - the observed erosion at ELM energies larger than $\sim 50 \mathrm{~kJ}$ is much larger and can be described with an Arrhenius-type fit ${ }^{51}$. Also chemical erosion should be smaller than $\sim 0.1 \%$ according to large surface temperatures expected during an ELM. Possible explanation is a decomposition of formerly deposited carbon layers under ELM impact. This is in line with the observation, that bare graphite material does not suffer from enhanced erosion, as observed in the outer divertor of JET where no layers are formed.
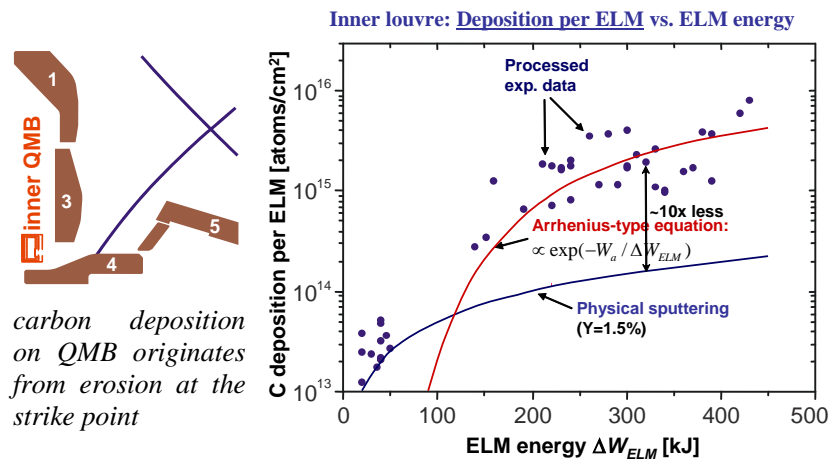

Figure 17: Influence of ELM energy on erosion of carbon layers in the inner divertor of JET ${ }^{51}$.

\section{Erosion of activated materials}

In contrast to present fusion experiments, ITER will produce significant fluxes of high energetic neutrons to the first wall elements during D-T operation, leading to material damages. Whereas physical and chemical sputtering of non-irradiated materials has been investigated intensively, plasma-wall interaction at neutron damaged materials has been analyzed in much less detail. Main effects of fusion neutron (14.1 MeV) irradiation in fusion devices are the production of radiation-induced defects (such as vacancies, interstitials or traps), changes of the microstructure and change of chemical composition due to transmutation. These processes can lead to degradation of the irradiated materials. For instance, thermal conductivity and ductility can decrease. Moreover, swelling and $\mathrm{He}$ embrittlement can occur. Hydrogen diffusion, trapping and recycling will be influenced by radiation damages and thus finally strongly determine bulk retention of fuel. However, in the following only possible influence of radiation damage on erosion properties of materials is discussed.

The effect of radiation damage on sputtering has been investigated in the linear plasma simulator LENTA 52 . Instead of radiation damage due to fusion neutrons, energetic ions have been used to produce radiation damages in the materials. Carbon-based materials have been bombarded with $5 \mathrm{MeV} \mathrm{C}^{+}$ions. Average produced damage is calculated (SRIM, a BCA code similar to TRIM) to be $\langle\mathrm{D}\rangle=9.7 \mathrm{dpa}$ with maximum damage of $\mathrm{D}$ $=60 \mathrm{dpa}$ at $5 \mu \mathrm{m}$ inside the sample. Various types of graphite have been irradiated and then exposed to the linear plasma device LENTA with $\mathrm{D}^{+}$impact energy of $100 \mathrm{eV}$ and surface temperature less than $40^{\circ} \mathrm{C}$. Erosion has been measured by means of weight loss. Enhanced erosion of irradiated samples compared to non-irradiated ones has been observed as following:

$-Y_{\mathrm{SEP} \text { irr }} / \mathrm{Y}_{\mathrm{SEP}}=2.6$
$-\mathrm{Y}_{\text {pyro irr }} / \mathrm{Y}_{\text {pyro }}=4.8$
$-\mathrm{Y}_{\mathrm{MPG} \text { irr }} / \mathrm{Y}_{\mathrm{MPG}}=1.6$

Tungsten has been bombarded with 3-4 $\mathrm{MeV} \mathrm{He}^{2+}$ ions to create radiation damages. SRIM calculations reveal maximum damage of $\mathrm{D}=5 \mathrm{dpa}$ at a depth of 6 $\mu \mathrm{m},\langle\mathrm{D}\rangle=0.3 \mathrm{dpa}$. Exposure of irradiated tungsten samples to the LENTA plasma did show - in contrast to carbon - no clear effect of radiation damage on the erosion.

However, experiments with fusion relevant plasmafacing materials (including also beryllium) having neutron-induced damages are missing. Damage profiles induced by fast neutrons from fusion may be different from ion-induced ones (e.g. due to broader energy spectrum compared to monoenergetic ions) and thus leading to different effects.

\section{SUMMARY \& CONCLUSIONS}

The most important sputtering and erosion mechanisms occurring in fusion experiments have been described. Physical sputtering occurs for all combinations of projectile and target but disappears at low impact energies below a threshold (around several $\mathrm{eV}$ ). Eroded species are mainly neutral atoms or small clusters from the substrate material. Under most 
conditions physical sputtering can be described by collision cascades inside the solid initiated by the impinging projectile using the binary collision approximation. However, at low impact energies $(<\sim 10$ $\mathrm{eV}$ ) molecular dynamics methods have to be used to take into account chemical effects. The sputtering yield for high- $Z$ materials is in general smaller than for low-Z materials. Chemical erosion and sputtering occurs only for special combinations of projectile and target material. In fusion research chemical erosion/sputtering due to hydrogen (and its isotopes) is of main importance for carbon-based materials and beryllium. For carbon also the erosion due to oxygen is relevant. Eroded species are molecules formed out of projectile and carbon - thus hydrocarbons $\mathrm{C}_{\mathrm{x}} \mathrm{H}_{\mathrm{y}}, \mathrm{BeH}$ and $\mathrm{BeH}_{2}$ and $\mathrm{CO}_{\mathrm{x}}$. In contrast to physical sputtering no threshold energy exists. At large surface temperatures and high incoming fluxes the yield of chemical erosion/sputtering for carbon decreases significantly. A model, which describes the thermodynamical and kinetic processes involved in chemical erosion and sputtering for carbon has been presented.

Main features of backscattering of atomic species have been described. As for physical sputtering, the underlying physics can be described with the binary collision approximation or molecular dynamic simulations depending on the impact energy. Sticking of hydrocarbons is rather complex. Experimental data and also molecular dynamics simulations exist for various hydrocarbons species. It is seen that particularly unsaturated hydrocarbons contribute to layer growth.

Fuel retention, which is a major concern in future fusion devices, takes place by means of adsorption, implantation, bulk diffusion and co-deposition. From present experiments it is concluded that long-term retention in devices with low $\mathrm{Z}$ first walls (e.g. $\mathrm{C}$ or $\mathrm{Be}$ ) will be dominated by co-deposition of fuel in deposited layers. It is thus important to understand the involved processes of erosion, material transport and deposition.

Examples of erosion and deposition experiments in fusion devices and plasma simulators have been given. The main dependencies of physical sputtering, chemical erosion and sputtering are confirmed by experimental observations. However, in fusion experiments the situation is more complicated due to the presence of various species, which leads to material mixing.

\section{REFERENCES}

1 Progress in the ITER Physics Basis, Nucl. Fusion 47 (2007).

2 G. COUNSELL et al., "Tritium retention in next step devices and the requirements for mitigation and removal techniques," Plasma Phys. Control. Fusion, 48, No. 12 B, B189-B199 (2006).

3 A. KIRSCHNER et al., "Estimations of erosion fluxes, material deposition and tritium retention in the divertor of ITER," J. Nucl. Mat., 390-391, 152 (2009).
4 R. BEHRISCH (ed.), "Sputtering by particle bombardment. I. Physical sputtering of singleelement solids, “ Topics in applied physics, Vol. 47, Berlin/Heidelberg/New York, Springer 1981.

5 J. BOHDANSKY, "A universal relation for the sputtering yield of monoatomic solids at normal ion incidence," Nucl. Instr. Meth., B2, 587 (1984) and C. GARCIA-ROSALES et al., "Revised formulae for sputtering data," J. Nucl. Mat., 218, 8 (1994).

6 W. ECKSTEIN, "Calculated sputtering, reflection and range values," Report IPP, 9/132 (2002).

7 B. UNTERBERG, theses proceedings.

8 M. KÜSTNER, W. ECKSTEIN, E. HECHTL, J. ROTH, "Angular dependence of the sputtering yield of rough beryllium surfaces," J. Nucl. Mat., 265, 22 (1999).

9 H.L. BAY, W. BERRES, E. HINTZ, "Surface normal velocity distribution of sputtered $\mathrm{Zr}$-atoms for light-ion irradiation," Nucl. Instr. Meth., 194, 555 (1982).

10 J. BOHDANSKY, "Sputtering," in Data compendium for plasma-surface interactions, $\mathrm{Nucl}$. Fusion, Special Issue, eds. R.A. Langley et al. (1984).

11 R.K. JANEV, Yu.V. RALCHENKO, T. KENMOTSU, K. HOSAKA, "Unified analytic representation of physical sputtering yield," J. Nucl. Mat., 290-293, 104 (2001).

12 Y. YAMAMURA, Y. ITIKAWA, N. ITOH, "Angular dependence of sputtering yields of monoatomic solids," Japan Report, IPPJ-AM-26, Nagoya University (1983).

13 W. ECKSTEIN, Computer simulation of ion-solid interaction, Springer, Berlin (1991).

14 W. ECKSTEIN, R. DOHMEN, A. MUTZKE, R. SCHNEIDER, "SDTrimSP: A Monte-Carlo code for calculating collision phenomena in randomized targets," Report IPP, 12/3 (2007).

15 W.D. WILSON, L.G. HAGGMARK, J.P. BIERSACK, "Calculations of nuclear stopping, ranges, and straggling in the low-energy region," Phys. Rev., B 15, 2458 (1977).

16 W. ECKSTEIN, W. MÖLLER, “Computer simulation of preferential sputtering," Nucl. Instr. Meth. Phys. Research, B7/8, 727 (1985).

17 W. ECKSTEIN, "Oscillations of sputtering yield," Nucl. Instr. Meth. Phys. Research, B171, 435 (2000).

18 J. KÜPPERS, "The hydrogen surface chemistry of carbon as a plasma facing material," Surf. Sci. Rep., 22, 249 (1995).

19 E. VIETZKE et al., "Chemical erosion of amorphous hydrogenated carbon films by atomic and energetic hydrogen", J. Nucl. Mat., 145-147, 443 (1987).

20 J. ROTH, "Chemical erosion of carbon based materials in fusion devices," J. Nucl. Mat., 266-269, 51 (1999). 
21 J. ROTH et al., "Flux dependence of carbon chemical erosion by deuterium ions," Nucl. Fusion, 44, L21-L25 (2004).

22 E. VIETZKE, K. FLASKAMP, V. PHILIPPS, "Hydrocarbon formation in the reaction of atomic hydrogen with pyrolytic graphite and the synergistic effect of argon ion bombardment," J. Nucl. Mat., 111+112, 763 (1982).

23 E. VIETZKE, V. PHILIPPS, K. FLASKAMP, Chemical reactivity of atomic hydrogen on graphite pre-irradiated by hydrogen and argon ions," J. Nucl. Mat., 162-164, 898 (1989).

24 Y. UEDA, K. TOBITA, Y. KATOH, "PSI issues at plasma facing surfaces of blankets in fusion reactors," J. Nucl. Mat., 313-316, 32 (2003).

25 T. SHIMIDA et al., "Blister formation on tungsten surface by irradiating hydrogen and carbon mixed ion beam," J. Plasma and Fus. Research, 78, 289 (2002).

26 V. PHILIPPS, K. FLASKAMP, E. VIETZKE, "Enhancement of the sputtering yield of pyrolytic graphite at elevated temperatures," J. Nucl. Mat., 111\&112, 781 (1982).

27 V. PHILIPPS et al., "Investigation of radiation enhanced sublimation of graphite test-limiters in TEXTOR," J. Nucl. Mat., 220-222, 467 (1995).

28 D.A. ALMAN, D.N. RUZIC, "Molecular dynamics simulation of carbon/hydrocarbon reflection coefficients on a hydrogenated graphite surface," J. Nucl. Mat., 313-316, 182 (2003).

29 K. OHYA et al., "Simulation of hydrocarbon reflection from carbon and tungsten surfaces and its impact on codeposition patterns on plasma facing components," J. Nucl. Mat., 390-391, 72 (2009).

30 W. ECKSTEIN, H. VERBEEK, "Data on light ion reflection," Report IPP, 9/32 (1979).

31 I. BIZYUKOV et al., "Relevance of surface roughness to tungsten sputtering and carbon implantation," J. Appl. Phys., 100, 113302 (2006).

32 M. VAN SCHOOR, these proceedings.

33 C. HOPF et al., "Surface loss probabilities of hydrocarbon radicals on amorphous hydrogenated carbon film surfaces," J. Appl. Phys., 87, 2719 (2000).

34 M. MEIER, A. VON KEUDELL, W. JACOB, "Consequences of the temperature and flux dependent sticking coefficient of methyl radicals for nuclear fusion experiments," Nucl. Fus., 43, 25 (2003).

35 A. VON KEUDELL, W. JACOB, "Elementary processes in plasma-surface interaction: $\mathrm{H}$-atom and ion-induced chemisorption of methyl on hydrocarbon film surfaces," Progress in Surf. Science, 76, 21 (2004).

36 D. A. ALMAN, D. N. RUZIC, "Molecular dynamics simulation of hydrocarbon reflection and dissociation coefficients from fusion-relevant carbon surfaces," Phys. Scri., T111, 145 (2004).
37 H. TOYODA, H. KOJIMA, H. SUGAI, "Mass spectroscopic investigation of the $\mathrm{CH}_{3}$ radicals in a methane rf discharge," Appl. Phys. Lett., 54, 1507 (1989).

38 M. SHIRATANI et al., "Surface reaction kinetics of $\mathrm{CH}_{3}$ in $\mathrm{CH}_{4}$ rf discharge studied by time-resolved threshold ionization mass spectrometry," Jap. J. Appl. Phys., 36, 4752 (1997).

39 P. TRÄSKELIN et al., "Molecular dynamics simulations of $\mathrm{CH}_{3}$ sticking on carbon first wall structures," J. Nucl. Mat., 313-316, 52 (2003).

$40 \quad$ E. NEYTS et al., "Unraveling the deposition mechanism in a-C:H thin-film growth: A moleculardynamics study for the reaction behavior of $\mathrm{C}_{3}$ and $\mathrm{C}_{3} \mathrm{H}$ radicals with a-C:H surfaces," L. Appl. Phys., 99, 014902 (2006).

41 A. KRETER, these proceedings.

42 U. SAMM, "TEXTOR: A pioneering device for new concepts in plasma-wall interaction, exhaust, and confinement," in Special Issue on TEXTOR, Fusion Science Techn., 47, 73 (2005).

43 A. POSPIESZCZYK et al., "Chemical erosion measurements from various carbon based limiters and coatings from TEXTOR-94," J. Nucl. Mat., 241-243, 833 (1997).

44 A. KRETER et al., "Investigation of carbon transport by ${ }^{13} \mathrm{CH}_{4}$ injection through graphite and tungsten test limiters in TEXTOR," Plasma Phys. Control. Fus., 48, 1401 (2006).

45 A. KRETER et al., "Study of local carbon transport on graphite, tungsten and molybdenum test limiters in TEXTOR by ${ }^{13} \mathrm{CH}_{4}$ tracer injection," J. Nucl. Mat., 363-365, 179 (2007).

46 A. KIRSCHNER et al., "Simulation of the plasmawall interaction in a tokamak with the Monte-Carlo code ERO-TEXTOR," Nucl. Fus. 40, 989 (2000).

47 A. KIRSCHNER et al., "Modelling of carbon transport in fusion devices: evidence of enhanced reerosion of in-situ re-deposited carbon," J. Nucl. Mat., 328, 62 (2004).

48 S. DROSTE et al., "Modelling of ${ }^{13} \mathrm{CH}_{4}$ injection experiments with graphite and tungsten test limiters in TEXTOR using the coupled code EROSDTrimsP," Plasma Phys. Control. Fus. 50, 015006 (2008).

49 V. PHILIPPS et al., "Experiments with tungsten limiters in TEXTOR-94," J. Nucl. Mat., 258, 858 (1998).

50 G. SERGIENKO et al., "Erosion of a tungsten limiter under high heat flux in TEXTOR," J. Nucl. Mat., 363-365, 96 (2007).

51 A. KRETER et al., "Nonlinear impact of edge localized modes on carbon erosion in the divertor of the JET tokamak," PRL, 102, 045007 (2009).

52 B.I. KHRIPUNOV et al., "Evidence of radiation damage impact on material erosion in plasma environment," J. Nucl. Mat., 390-391, 921 (2009). 